ISSN: $1130-3743$ - e-ISSN: 2386-5660

DOI: http://dx.doi.org/10.14201/teoredu2924159

\title{
EFECTOS DE LA COMUNICACIÓN DIALÓGICA EN LA MEJORA DE LA COMPOSICIÓN ESCRITA EN ESTUDIANTES DE PRIMARIA
}

\author{
Effects of communication in improving dialogic written \\ in primary students composition
}

\section{Des effets de la communication dialogique dans les améliorations de la composition écrite chez étudiants de primaire}

Raúl GuTiÉRrez-Fresneda y Antonio DíEz-Mediavilla

Universidad de Alicante. Facultad de Educación. Departamento de Innovación y Formación Didáctica. Carretera de San Vicente del Raspeig, s/n. 03690 Alicante. raul.gutierrez@ua.es; antonio.diez@ua.es

Fecha de recepción: enero de 2017

Fecha de aceptación: marzo de 2017

RESUMEN

La escritura es una herramienta imprescindible para el éxito académico que requiere de unas habilidades y estrategias de gran complejidad que no siempre se enseñan en el ámbito escolar. El propósito de este trabajo fue analizar si, mediante un programa de enseñanza de las estrategias de producción escrita implementado a través de la comunicación dialógica en grupos interactivos, se adquiere una mejor composición escrita. Se empleó un diseño cuasiexperimental de comparación entre grupos con medidas pretest y postest. En el estudio participaron 409 estudiantes con edades comprendidas entre los 8 y los 9 años. Los resultados apoyan el valor potencial del programa y sugieren el desarrollo de modelos de enseñanza que integren prácticas de comunicación dialógica, por lo que se recomienda el diseño 
de programas orientados al desarrollo de las estrategias de composición a través de grupos interactivos en cuanto que facilitan el aprendizaje de la composición escrita.

Palabras clave: lenguaje hablado; escritura; expresión escrita; lenguaje escrito; producción de textos.

\section{SUMMARY}

Writing is an essential tool for academic success, requiring skills and strategies of great complexity that are not always taught in the school environment. The purpose of this study was to analyse whether, using a program of teaching strategies of written production implemented through dialogic communication in interactive groups, improved the acquisition of written composition. A quasi-experimental design of comparison between groups with pre-test and post-test measurements was used. 409 students between the ages of 8 and 9 participated in the study. The results support the potential value of the program and suggest the development of teaching models that integrate dialogic communication practices. Therefore, the design of programs oriented towards the development of composition strategies through interactive groups that facilitate the learning of written composition is recommended.

Key words: spoken language; writing; written expression; written language; text production.

\section{SOMMAIRE}

L'écriture est un outil indispensable pour le succès académique qu'il requiert de quelque habileté et des stratégies de grande complexité qui sont rarement apprises dans le domaine scolaire. Le propos de ce travail a été d'analyser si, au moyen d'un programme d'enseignement des stratégies de production écrite mis en application à travers de la communication dialogique dans des groupes interactifs, une meilleure composition écrite est acquise. Un dessin quasi-expérimental de comparaison s'est employé entre des groupes avec mesures pretest et postest. À l'étude 409 étudiants ont participé avec âges compris entre 8 et 9 ans. Les résultats pèsent la valeur potentielle du programme et appuient le développement de modèles d'enseignement qui intègrent des pratiques de communication dialogique de ce qui se suggère le dessin de programmes orientés au développement des stratégies de composition à travers des groupes interactifs aussitôt que qu'ils facilitent l'apprentissage de la composition écrite.

Mots clés: un langage parlé; une écriture; une expression écrite; un langage écrit; une production de textes. 
RAÚl GUTIÉRREZ-FRESNEDA Y ANTONIO DÍEZ-MEDIAVILLA

EFECTOS DE LA COMUNICACIÓN DIALÓGICA EN LA MEJORA DE LA COMPOSICIÓN ESCRITA...

\section{INTRODUCCIÓN}

La composición escrita se ha convertido en uno de los principales referentes de estudio en el ámbito educativo tanto por la complejidad de los procesos y habilidades implicados como por la importancia que presenta en la adquisición de los contenidos escolares.

La escritura constituye una actividad compleja que precisa del dominio de múltiples habilidades y de la capacidad de quien escribe para coordinar diversas acciones. Se entiende como un proceso de comunicación que requiere generar y organizar las ideas para desarrollarlas en un contexto con una intención comunicativa determinada.

Durante los últimos años han sido varios los planteamientos que han intentado explicar el desarrollo de la composición escrita, los cuales se agrupan en tres grandes modelos (Castelló y Monereo, 1996; Salvador, 2008). El enfoque más tradicional es el modelo orientado al producto, caracterizado por el análisis de la expresión escrita desde una perspectiva puramente lingüística, dando valor a los aspectos formales y estructurales del texto (grafía, ortografía, significado y cohesión textual). Las limitaciones a la hora de explicar los procesos que subyacen a la actividad de escritura contribuyeron a desplazar el interés de la investigación sobre este enfoque hacia los modelos orientados al proceso, de orientación cognitiva y predominantes en la actualidad, los cuales atienden a la secuencia que sigue el escritor al elaborar un texto. Finalmente, el modelo contextual o ecológico complementa las aportaciones de los modelos cognitivos profundizando en la dimensión social y comunicativa de la escritura, considerando la composición escrita como un proceso comunicativo y social que adquiere sentido en el contexto social y cultural en el que se desarrolla.

De esta complejidad de la escritura surgen diferentes modelos teóricos para la enseñanza de la composición escrita. Entre estos destaca el modelo cognitivo de Flower y Hayes (1981), posteriormente modificado y actualizado por Hayes (1996), el cual es considerado actualmente como el modelo que mejor explica la escritura de textos, al concebir esta como un proceso de resolución de problemas en el que el escritor activa estrategias de planificación, análisis e inferencia. Para dichos autores la escritura requiere no sólo la intervención de procesos cognitivos de bajo nivel para la traducción gráfica, sino también la activación y coordinación de procesos cognitivos de alto nivel: planificación, producción y revisión, regulados por otros de carácter metacognitivo que se ponen en práctica para elaborar la producción de un texto.

La planificación es un proceso mental referente al diseño previo del escritor sobre lo que quiere decir y cómo lo va a decir. En esta fase se establecen los objetivos del texto y el plan global que orienta la producción textual teniendo en consideración las características del tema y la audiencia a la que se dirige. Incluye varios subprocesos u operaciones cognitivas: la génesis de ideas o contenidos, que supone la búsqueda de información en la memoria a largo plazo; la organización de la información en una estructura global que da coherencia al texto escrito, y 
el establecimiento de objetivos que guían y controlan el acto y el producto de la composición.

La trascripción o producción del texto supone la transformación de las ideas del escritor en lenguaje inteligible para el lector. Es un proceso muy complejo, ya que el escritor debe atender a varias demandas: los propósitos y el contenido del texto, la construcción morfosintáctica, la ejecución gráfica de las letras, la adecuación ortográfica... El propósito comunicativo y los procesos que se desarrollan están influidos por dos tipos de condicionantes: procedimentales, referentes a cómo realizar el proceso de escritura, y de contenido, que especifican todo lo que el escritor quiere decir a sus lectores.

La revisión es un proceso cognitivo en el que el escritor analiza y corrige la composición textual comparándola con la representación mental que se va elaborando, tanto desde el punto de vista formal como conceptual y funcional. La revisión incluye dos subprocesos: la evaluación del resultado y la modificación y corrección del texto producido. La función de evaluación es fundamental para identificar y analizar los problemas del texto (Hayes, 1996).

El interés de la investigación sobre cómo mejorar los procesos de composición se ha incrementado sustancialmente debido al elevado porcentaje de estudiantes que presentan dificultades para escribir textos coherentes, organizados y adaptados a distintas situaciones comunicativas (Bereiter y Scardamalia, 1987; González, 2003; Troia, 2006; Watanabe y Hall-Kenyon, 2011; Kiuhara, O’Neill, Hawken y Graham, 2012).

La importancia que tiene la adquisición de un buen proceso de aprendizaje de la escritura en el ámbito educativo es evidente. Sin embargo, a pesar de las aportaciones de los estudios realizados en los últimos años se sigue encontrando un gran número de alumnos que presentan carencias en el dominio de esta importante habilidad lingüística (OCDE, 2013). Uno de los motivos puede deberse a que los trabajos efectuados sobre la composición escrita han estado centrados mayoritariamente en analizar los procesos cognitivos que el escritor pone en marcha al elaborar su producción textual, así como en los diferentes subprocesos implicados (selección de objetivos, génesis de ideas, organización y estructuración del contenido...) atendiendo al proceso de elaboración de la escritura de manera individual. Es decir, existe la tendencia de considerar al escritor como un sujeto que afronta la elaboración del texto a partir de sus esquemas de conocimiento y de las acciones estratégicas que lleva a cabo (Flowers y Hayes, 1981; Hayes, 1986; García y Marbán, 2003; Arroyo y Salvador, 2005; Gallego y Mendías, 2012) sin explotar las posibilidades que conlleva la utilización de las estrategias de composición textual desde un plano dialógico.

Existe mucha investigación en la que se analiza el proceso de composición de la escritura que los estudiantes realizan a nivel particular para dar respuesta a las demandas de elaboración de diferentes textos, lo que nos ha permitido conocer cuáles son los aspectos en los que los alumnos encuentran mayores dificultades de expresión escrita (Dockrell, Lindsay, Connelly y Mackie, 2007; González y Martín, 
2006; Gallego y Mendías, 2012; Cabero et al., 2015). De igual modo, se sabe qué operaciones ponen en práctica los estudiantes durante las distintas fases de producción textual (planificación, traducción y revisión) y las carencias existentes durante su desarrollo (Arroyo y Salvador, 2005; Gallego, 2008; Salvador y García, 2009; Gutiérrez, 2014; Espino, 2015). Sin embargo, son escasos los estudios que analizan la potencialidad que ofrece el hecho de hacer explícitas las estrategias que los estudiantes ponen en práctica para construir el significado del texto a través de situaciones de escritura colaborativa (Guzmán y Rojas-Drummond, 2012).

En trabajos anteriores se ha demostrado que cuando los estudiantes colaboran de manera conjunta con otros compañeros llegan a elaborar mejores composiciones textuales (sobre todo respecto al vocabulario utilizado), en comparación con los textos producidos individualmente (Yarrow y Topping, 2001). Los intercambios verbales de información respaldan la idea de que las actividades compartidas generan mayores procesos de reflexión personal y grupal derivados de las sugerencias recibidas por los propios alumnos (Montanero, Lucero y Fernández, 2014). Los efectos positivos de la ayuda compartida también han sido observados en conductas estratégicas en la composición de textos narrativos y argumentativos (Harris, Graham y Mason, 2006).

En este sentido, potenciar la relación entre los procesos de oralidad y escritura de manera compartida a través del aprendizaje estratégico de composición escrita puede contribuir a que los alumnos se vayan apropiando de las habilidades necesarias para producir textos de mayor calidad de forma individual, autorregulada y autónoma. En este sentido la verbalización de las estrategias de composición a través de la formación de grupos interactivos en los que se facilite la puesta en común de las aportaciones de los distintos compañeros a través de la reflexión individual y colectiva puede resultar una medida eficaz para la adquisición de estrategias de aprendizaje de la escritura.

Desde la perspectiva social, el logro de las metas que se propone cada persona en un trabajo cooperativo tiene una correlación positiva con el logro de los objetivos del resto de los componentes del grupo, lo que se traduce en la unión de intereses, capacidades, recursos, estrategias... para la consecución del objetivo pretendido. Desde este planteamiento, la escritura supone una producción compartida que promueve la reflexión crítica, lo que puede favorecer la toma de decisiones sobre qué estrategias aplicar, cómo aplicarlas, cuándo aplicar cada una de ellas..., conocimiento que puede contribuir a la mejora del proceso de composición en las distintas situaciones comunicativas.

El objetivo de este estudio fue analizar el efecto que la intervención de un programa centrado en el desarrollo de las estrategias de composición escrita basado en la comunicación dialógica presenta en la adquisición de la producción textual. Para ello, se compara el grado de adquisición de la composición de la escritura en dos grupos de estudiantes de entre 8 y 9 años, uno que recibe intervención en el proceso de expresión escrita mediante la instrucción en estrategias de composición en grupos interactivos y otro que sigue el mismo programa de enseñanza de 
composición escrita, pero a través de la clase magistral tradicional. La hipótesis que se plantea es que el alumnado perteneciente al grupo que recibe instrucción de la composición escrita mediante situaciones de comunicación dialógica incrementará las habilidades de composición de textos.

\section{MÉTODO}

\subsection{Participantes}

Para la selección de los participantes se tuvieron en cuenta tanto la heterogeneidad de los centros (público y concertado) como el hecho de que estos estuvieran ubicados en un contexto sociocultural de nivel medio. En el estudio se contó con 409 participantes con edades comprendidas entre los 8 y los 9 años $(M=8.52 ; D T=0.54)$, de los cuales el $49.3 \%$ eran niños y el $50.7 \%$ niñas. Estos estudiantes pertenecían a cuatro centros educativos públicos y concertados, de los cuales dos colegios (uno público y otro concertado) fueron asignados al grupo experimental (206 alumnos) y los otros dos (público y concertado) al grupo control (203 alumnos). De los 206 participantes experimentales, el 48.6\% son varones y el 51.4\% mujeres, mientras que de los 203 participantes del grupo control, el 49.2\% son varones y el 50.8\% son mujeres. El análisis de contingencia (chi cuadrado de Pearson) entre condición y sexo no evidenció diferencias estadísticamente significativas $\left(\mathrm{X}^{2}=0.56, p>.05\right)$.

\subsection{Instrumentos de evaluación}

Con la finalidad de evaluar la variable dependiente objeto de estudio se utilizaron tres instrumentos de evaluación con garantías psicométricas de fiabilidad y validez.

- Batería de Evaluación Cognitiva de la Lectura y Escritura (BECOLE) (Galve, 2005). Evalúa los principales procesos implicados en la escritura a nivel léxico, sintáctico-semántico oracional y textual. En este estudio se utilizó la prueba de ordenación de palabras para formar una oración y la prueba de construcción de estructuras sintácticas que evalúan el nivel sintáctico-semántico oracional. En todas las tareas se concede un punto por acierto. Dicha prueba presenta un coeficiente de fiabilidad de Cronbach de 0.95. Además con los datos del presente estudio a través del índice de fiabilidad compuesta $(\mathrm{FC}=.84)$ y la varianza media extractada $(\mathrm{VmE}=72.46 \%$ ) es posible comprobar que la prueba es fiable.

- Proescri-Primaria (Prueba de evaluación de los procesos cognitivos en la escritura) (Artiles y Jiménez, 2007). Se utilizaron las pruebas relativas al dominio de los procesos de estructuración léxica, los procesos de estructuración morfosintáctica y los procesos de planificación. En cada una de estas pruebas se concede un 
punto por cada respuesta correcta. De acuerdo con el procedimiento de Cronbach el coeficiente de fiabilidad es de 0.85. Además se calculó la fiabilidad compuesta (FC) y la varianza media extractada (VME) con los datos del presente estudio. Los resultados mostraron que la fiabilidad fue elevada $(\mathrm{FC}=0.83)$, y la varianza media extractada fue superior a $0.50(\mathrm{vmE}=56.73 \%)$ lo que implica que un alto porcentaje de la varianza es explicada por el constructo.

- PROESC. Evaluación de los procesos de escritura (Cuetos, Ramos y Ruano, 2003). Se utilizaron las pruebas de planificación de textos narrativos y expositivos mediante la escritura de un cuento y la redacción sobre un animal conocido. En el texto narrativo se valoraron tanto los contenidos como la coherencia-estilo, mientras que en el texto expositivo se evaluaron los contenidos y la presentación. La puntuación máxima en cada apartado es de 10 puntos. Dicha prueba presenta un coeficiente de fiabilidad de Cronbach de 0.82. Además, con los datos del presente estudio a través del índice de fiabilidad compuesta $(\mathrm{FC}=0.87)$ y la varianza media extractada $(\mathrm{VME}=56.62 \%)$ es posible comprobar que la prueba es fiable.

\subsection{Diseño y procedimiento}

El estudio utilizó un diseño cuasiexperimental de medidas repetidas pretestpostest con grupo control. Antes y después de implementar el programa de intervención se aplicaron los tres instrumentos de evaluación descritos anteriormente a los participantes experimentales y control con la finalidad de medir la variable dependiente sobre la que se hipotetizó que el programa iba a tener efecto en la composición escrita.

La evaluación inicial del alumnado se llevó a cabo de forma colectiva en el aula ordinaria en el mes de noviembre y dentro del horario escolar. Posteriormente, se implementó el programa de intervención durante 10 semanas (3 sesiones de 45 minutos semanalmente). Los alumnos experimentales estaban distribuidos en equipos interactivos y los del grupo control de manera individual según la estructura del aula tradicional. En el mes de marzo, momento en el que ya se había aplicado por completo el programa, se volvió a realizar la evaluación a todo el alumnado con los mismos instrumentos. El estudio respetó los valores éticos requeridos en la investigación con seres humanos (consentimiento informado, derecho a la información, protección de datos personales, garantías de confidencialidad, no discriminación, gratuidad y posibilidad de abandonar el programa en cualquiera de sus fases).

\subsection{Programa de intervención}

El programa para el aprendizaje de la composición escrita que se utilizó tenía como objetivo desarrollar de manera explícita y activa las estrategias cognitivas que permiten al escritor construir textos de manera significativa y funcional dando respuesta a diferentes situaciones comunicativas a través del desarrollo de los 
subprocesos implicados en la producción de textos: planificación, redacción y revisión.

En la primera sesión el docente leyó un texto narrativo y explicó la estructura textual que caracteriza a este tipo de escritos con la finalidad de que los estudiantes desarrollen determinadas estrategias que emplean los escritores y consideren previamente a la elaboración de la producción textual aspectos tales como los objetivos, la audiencia, el tema, así como el modo de tomar decisiones sobre la forma más adecuada de organizar las ideas. En la siguiente sesión se presentó un organizador gráfico con el propósito de desarrollar las estrategias de generación y organización de ideas. El proceso seguido consistió en la elección de un tema de entre varios presentados por el docente, analizar el modo de obtener información sobre esa temática, pensar en quiénes van a ser los destinatarios, determinar el objetivo de la composición y organizar las ideas de manera secuenciada.

Posteriormente, en una tercera sesión se trabajaron distintas estrategias de escritura de manera colectiva a partir de la información recogida en el organizador gráfico elaborado la sesión anterior con el propósito de explicitar los procesos que deben ponerse en práctica durante la elaboración de la composición: planificación, redacción y revisión de la producción textual. Con esta finalidad se trabajaron las siguientes estrategias:

Antes de escribir:

- Establecer el propósito de escritura (¿para qué vamos a escribir?).

- Activar conocimientos previos (¿qué sabemos de este tema?).

- Identificar la audiencia a la que se dirige (¿quién lo leerá?).

- Determinar el tipo de texto (¿qué tipo de texto vamos a escribir?).

- Generar ideas y organizarlas de manera secuenciada.

Durante la escritura:

- Emplear construcciones sintácticas correctas y variadas.

- Organizar las ideas en distintos párrafos.

- Utilizar la estructura textual adecuada según el objetivo de lectura.

- Usar correctamente la ortografía y los signos de puntuación.

- Emplear los mecanismos de cohesión y coherencia.

- Supervisar la propia composición.

Después de la escritura:

- Comprobar el texto producido con el plan previo.

- Revisar la organización y presentación del contenido.

- Corregir aspectos sintácticos, léxicos y ortográficos.

- Confirmar la consecución del objetivo de escritura establecido.

A partir de este trabajo, se implementó un modelo metodológico orientado a fomentar los procesos de comunicación entre distintos estudiantes. Con esta 
finalidad se constituyeron cinco grupos de carácter heterogéneo en relación a los diferentes ritmos y niveles de aprendizaje, habilidades sociales, género, liderazgo...

Cada uno de ellos estaba formado por cuatro o cinco participantes y dirigido por uno que actuaba como coordinador. Los responsables contaban con diferentes materiales de producción escrita que el profesor les había entregado y que previamente habían entrenado, los cuales estaban distribuidos en cinco sesiones y orientados al desarrollo de las siguientes estrategias:

- Análisis de los elementos de la situación de comunicación (escritor, audiencia, objetivo, tema).

- Generación y organización de ideas a través de organizadores visuales (mapas mentales, esquemas...).

- Selección de estructuras textuales diversas y reflexión sobre el modo de presentar la información.

- Exposición de las ideas en párrafos cuidando los mecanismos de cohesión y coherencia textual.

- Comparar las producciones textuales elaboradas con los propósitos iniciales establecidos atendiendo a todos los elementos de la situación comunicativa.

En la cuarta sesión cada grupo comenzó a trabajar con un coordinador las estrategias anteriores. Se comenzaba con un periodo de reflexión individual sobre las demandas de la tarea y posteriormente se iniciaba la puesta en común de manera dialógica. Mediante la exposición libre, cada componente del grupo realizaba aportaciones personales sobre las cuestiones y propuestas que se iban planteando en torno a los aspectos que caracterizan el modelo del buen escritor. Con la supervisión del profesor, se contribuía al desarrollo de las estrategias de trabajo encomendadas. En las siguientes sesiones los participantes de los grupos rotaron y pasaron a abordar otra estrategia, así hasta que todo el alumnado ejercitó las distintas estrategias de manera repetida hasta en cinco ocasiones. Se concluyó con una tarea de producción escrita conjunta del grupo clase en la que se pusieron de manifiesto las distintas estrategias que se habían trabajado en cada uno de los grupos interactivos, efectuándose una autoevaluación sobre el proceso realizado y los aprendizajes adquiridos. El grupo control recibió el mismo programa de enseñanza descrito, pero con la diferencia de que el alumnado trabajaba de manera individualizada siguiendo el modelo magistral tradicional. En ambos grupos el trabajo estuvo supervisado por los responsables del estudio semanalmente comprobando la adecuada realización de la planificación establecida.

\section{Resultados}

Con la finalidad de analizar el cambio en las variables objeto de estudio se realizaron análisis descriptivos (medias y desviaciones típicas) con las puntuaciones 
obtenidas en los test administrados en la fase pretest, postest y en la diferencia postest-pretest, así como análisis de varianza con las puntuaciones pretest (MANOvAs, ANOVAs) y análisis de covarianza (MANCOVAs, ANCOVAs) de las diferencias postestpretest en experimentales y control en las variables medidas antes y después de la intervención. Estos análisis se llevaron a cabo con el programa spss 20.0. Además, se calculó el tamaño del efecto (d de Cohen) (pequeño < 0.50; moderado 0.50-0.79; grande $\geq 0.80$ ). Los resultados del MANOva pretest para el conjunto de variables pusieron de relieve que antes de la intervención no existían diferencias significativas entre experimentales y control, $F(1,116)=1.24, p>.05$. Sin embargo, los resultados del MANCOVA de las diferencias postest-pretest, usando las puntuaciones pretest como covariables, fueron significativos $F(1,116)=2.48, p<.05$. Estos datos ponen de manifiesto que el programa de intervención tuvo un efecto significativo. Para analizar el cambio en cada variable se realizaron análisis descriptivos y de varianza que se presentan en la Tabla 1.

\subsection{Cambios en la composición a nivel sintáctico-semántico oracional}

Con el objeto de analizar la eficacia del programa en el desarrollo de la composición escrita a nivel sintáctico, se estudiaron los cambios en las puntuaciones obtenidas en el Test BECOLE. El MANOVA pretest no evidenció diferencias significativas entre experimentales y control, $F(1,116)=2.12, p>.05$, sin embargo, los resultados del MANCOVA postest-pretest, $F(1,116)=1.41, p<.05$, confirmaron diferencias significativas entre ambas condiciones. Respecto al análisis de cada variable de forma independiente en la ordenación de palabras no se produjeron diferencias entre los alumnos de los dos grupos, mientras que en la construcción de estructuras sintácticas sí que se observó un aumento mayor en los experimentales $(M=$ $0.64)$ que en el grupo control $(M=0.18)$. Los resultados del ANOva pretest pusieron de manifiesto que en esta fase no había diferencias significativas entre experimentales y control, $F(1,116)=.18, p>.05$. Sin embargo, el ANCOVA postest-pretest evidenció diferencias estadísticamente significativas entre condiciones, $F(1,116)=$ $4.53, p<.01$. El tamaño del efecto fue pequeño $(r=.33)$, lo que pone de relieve una mejora de la capacidad para elaborar la información escrita a nivel sintáctico atribuible al programa de intervención.

\subsection{Cambios en la composición morfosintáctica y en la semántica relacional}

Con el propósito de evaluar el impacto del programa en la comprensión morfosintáctica y en la semántica relacional, se analizaron los cambios en las puntuaciones obtenidas en el Test PROESCRI. El MANOVA pretest realizado con el conjunto de las tres variables medidas (estructura léxica, estructura morfosintáctica y planificación guiada) no evidenció diferencias significativas en la fase pretest entre experimentales y control, $F(1,116)=2.26, p>.05$. Sin embargo, se encontraron 
EFECTOS DE LA COMUNICACIÓN DIALÓGICA EN LA MEJORA DE LA COMPOSICIÓN ESCRITA..

diferencias significativas en el MANOVA postest-pretest, $F(1,116)=3.24, p<.01$, al igual que en el MANCOVA postest-pretest, $F(1,116)=4.12, p<.01$. Como se puede observar en la Tabla 1, no se produjeron diferencias en los estudiantes del grupo control y experimental en la estructuración léxica, pero sí en la variable estructura morfosintáctica, obteniendo la muestra experimental un incremento $(M=0.62)$, superior al conseguido por el grupo control $(M=0.22)$. Los resultados del ANOVA pretest pusieron de manifiesto que en esta fase no había diferencias significativas entre experimentales y control, $F(1,116)=.212, p>.05$. Sin embargo, los datos del ANCOVA de las diferencias postest-pretest evidenciaron resultados significativos, $F$ $(1,116)=8.43, p<.01$. El tamaño del efecto fue moderado $(r=0.54)$. En la variable planificación guiada se constatan de igual modo incrementos superiores en los experimentales $(M=0.70)$ frente a los del grupo control $(M=.28)$. Los resultados del ANOVA pretest evidenciaron que a priori no existían diferencias significativas entre ambas condiciones, $F(1,116)=0.258, p<.05$, realizándose un ANCOVA de las diferencias postest-pretest que indicó diferencias significativas, $F(1,116)=9.16$, $p<.001$, siendo el tamaño del efecto moderado $(r=0.61)$. Estos datos ponen de relieve una importante mejora del desarrollo de la construcción morfosintáctica y de la semántica relacional en estructuras textuales atribuible al programa de intervención.

\subsection{Cambios en los procesos de composición textual}

Para evaluar si el programa fue eficaz en el desarrollo de los procesos que intervienen en el aprendizaje de la composición escrita a nivel textual se analizaron los cambios en las puntuaciones logradas en el Test PROESC. El MANOVA pretest realizado para el conjunto de las variables de la prueba puso de manifiesto que no existían diferencias significativas en la fase pretest entre experimentales y control, $F(1,116)=1.26, p>.05$. Sin embargo, se encontraron diferencias significativas en el MANOVA postest-pretest, $F(1,116)=2.74, p<.01$, al igual que en el MANCOVA postest-pretest, $F(1,116)=3.42, p<.01$. Como se puede observar en la Tabla 1 , en la variable planificación de textos narrativos el grupo experimental obtiene una mejora $(M=0.70)$, mayor que la conseguida por el grupo control $(M=0.24)$. Los datos del ANCOVA de las diferencias postest-pretest indicaron resultados significativos, $F(1,116)=7.84, p<.001$. El tamaño del efecto fue moderado $(r=0.57)$. En la variable planificación de textos expositivos se constatan de igual modo incrementos superiores en los experimentales $(M=0.71)$ frente a los del grupo control $(M=$ 0.14). En el ANOva pretest los datos obtenidos pusieron de manifiesto que en esta fase no había diferencias significativas entre experimentales y control, $F(1,116)=$ $.326, p>.05$. No obstante, los datos del AnCova de las diferencias postest-pretest indicaron resultados significativos, $F(1,116)=5.46, p<.001$, siendo el tamaño del efecto moderado $(r=0.62)$. Estos datos ponen de manifiesto una mejora del aprendizaje de los procesos de composición escrita, tanto en estructuras narrativas como expositivas, atribuible al programa de intervención puesto en práctica. 
RAÚL GUTIÉRREZ-FRESNEDA Y ANTONIO DÍEZ-MEDIAVILLA

EFECTOS DE LA COMUNICACIÓN DIALÓGICA EN LA MEJORA DE LA COMPOSICIÓN ESCRITA...

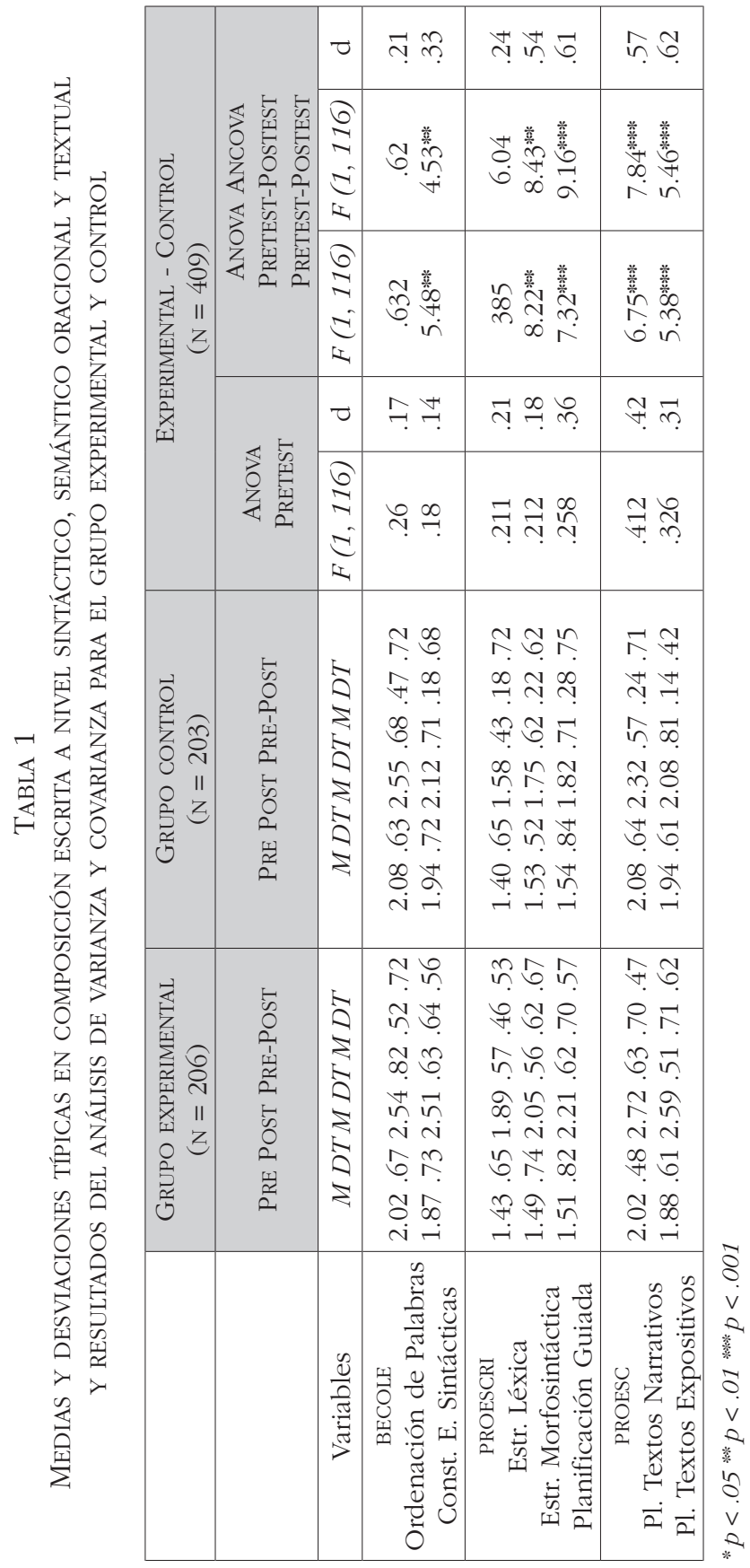


RAÚL GUTIÉRREZ-FRESNEDA Y ANTONIO DÍEZ-MEDIAVILLA

EFECTOS DE LA COMUNICACIÓN DIALÓGICA EN LA MEJORA DE LA COMPOSICIÓN ESCRITA..

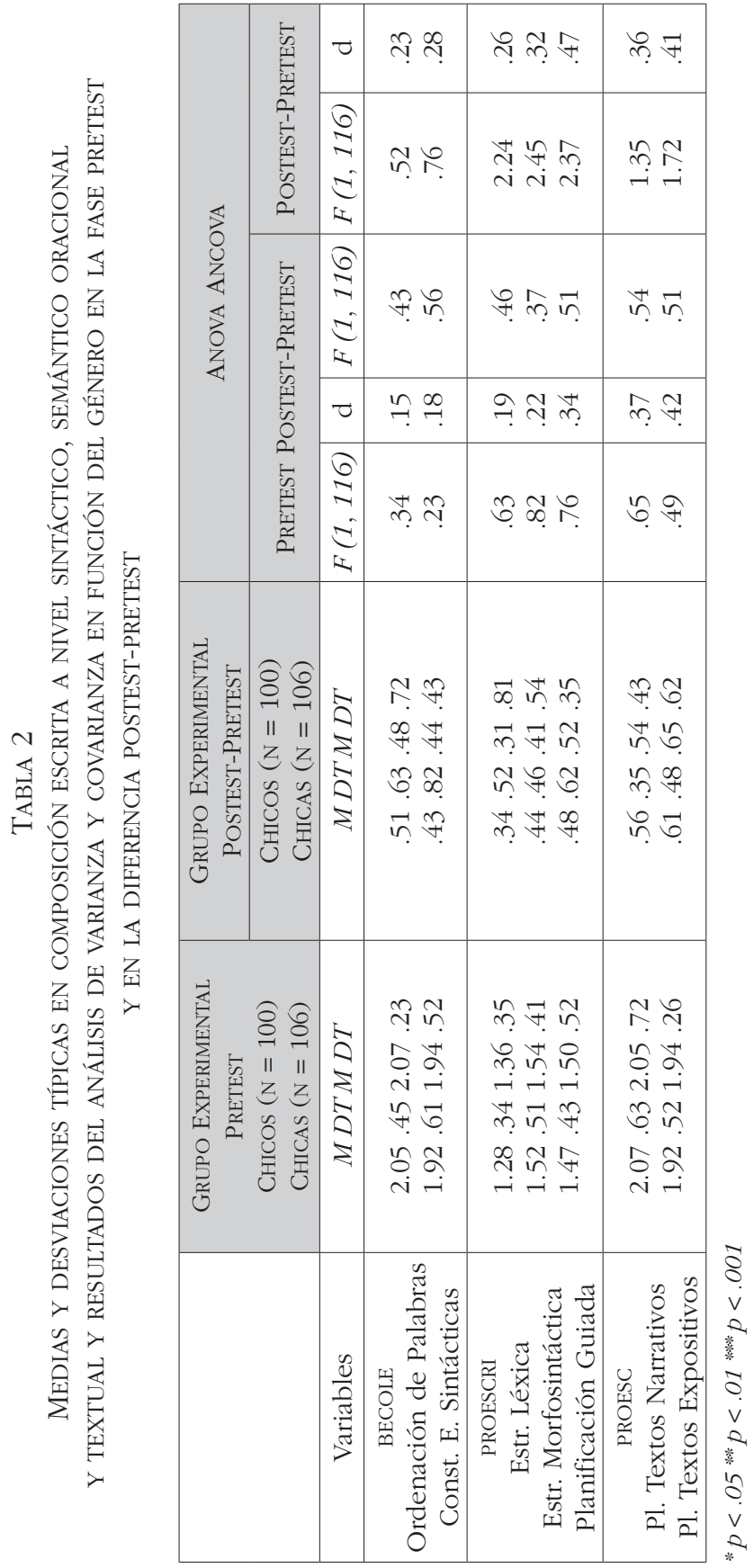




\subsection{Influencia del sexo en los efectos del programa}

Con la finalidad de explorar si el programa tuvo un efecto diferencial con respecto al sexo, es decir, si fue más beneficioso para los chicos o las chicas, o si el cambio producido por el programa era similar en ambos sexos se efectuaron análisis descriptivos (medias y desviaciones típicas) y de varianza (ANOVAS, ANCOVAS) en la fase pretest y en las diferencias postest-pretest en función del sexo. Los resultados señalan que en ninguna de las facetas estudiadas se produjeron efectos diferenciales del programa en función del sexo.

\section{DISCUSIÓN Y CONCLUSIONES}

El objetivo de este estudio era comprobar el efecto que la intervención de un programa orientado al desarrollo de las estrategias de producción del lenguaje escrito a través de la comunicación dialógica presenta en la mejora de la composición escrita. Los datos recogidos indican que la instrucción mediante grupos interactivos mejora de manera significativa el proceso de adquisición de la escritura. Una explicación de este hecho puede deberse a las contribuciones que la verbalización y la reflexión conjunta ofrecen a los aprendizajes personales, lo cual puede ser debido a que cuando los sujetos trabajan conjuntamente tienen más facilidades para aprender los unos de los otros e incorporar nuevas estrategias que contribuyan a mejorar sus propias producciones textuales. De hecho, son varios los estudios que han demostrado el efecto positivo de la reflexión compartida en el aprendizaje de los estudiantes (Quinton y Smallbone, 2010; Coulson y Harvey, 2013).

Analizando los diferentes niveles de producción escrita, se comprueba que el grupo experimental ha mejorado de manera significativa respecto al grupo control en la capacidad de composición a nivel sintáctico-semántico oracional, lo cual refleja que el programa implementado permite captar e integrar las relaciones sintagmáticas dentro del enunciado. Esto indica que la enseñanza de las estrategias que potencian la elaboración de estructuras sintácticas mediante la generación y organización de ideas favorece la elaboración del significado textual desde el nivel oracional.

También los estudiantes del grupo experimental lograron resultados mayores en la composición morfosintáctica y en la semántica relacional en la construcción de oraciones mediante la puesta en práctica de relaciones gramaticales. Se interpreta, por tanto, que la intervención implementada favoreció la capacidad de producción escrita desde los primeros niveles cognitivos de composición.

En relación a la composición semántica relacional, los datos obtenidos indican que los resultados del grupo experimental respecto al control son superiores en la capacidad para emplear de forma eficaz oraciones de diferente tipología respetando el orden lógico de los elementos que las integran. 
De igual modo, el programa de intervención contribuyó de manera relevante a la mejora de los distintos procesos de composición textual. En concreto, se produjeron logros significativos en la capacidad para aplicar técnicas de generación y organización de ideas; en la estructuración de la información en párrafos; en el uso de los mecanismos de coherencia y cohesión textual, y en los diferentes aspectos que contribuyen a incrementar la capacidad para planificar, organizar y elaborar el texto haciendo uso de los conocimientos previos en función de las demandas de la situación de comunicación. Se denota de igual forma un incremento en la capacidad de reflexión del alumnado participante en el programa y en una mejor organización general de los textos, lo que pone de manifiesto un mayor control y regulación del propio proceso de composición. Además, se producen mejoras en el grupo instruido en la capacidad para sintetizar, resumir y reordenar las ideas a partir de la información que se va elaborando, lo que puede ser debido a las aportaciones que ofrece la comunicación dialógica al planificar, redactar y revisar los escritos de manera conjunta y reflexiva.

Respecto al desarrollo de las habilidades metacognitivas de la escritura, los datos obtenidos por el grupo experimental evidencian las mejoras logradas en las distintas estrategias de aprendizaje, las cuales contribuyen a la toma de conciencia del proceso de composición. Al analizar los resultados se observa que el alumnado que ha participado en el programa organizado en equipos interactivos ha mejorado de manera significativa respecto al grupo control, entre otros aspectos, en la capacidad para definir los objetivos de escritura, atender a la audiencia, seleccionar la estructura textual más adecuada en función de las demandas de la tarea, generar ideas diversas y creativas, expresar y organizar la información de manera ordenada en diferentes párrafos, verificar la consecución de los objetivos establecidos durante la elaboración del texto y comparar al finalizar la composición producida con los planes establecidos.

En definitiva, los resultados analizados en su conjunto en los diferentes niveles de composición escrita constatan los efectos positivos del programa favoreciendo la adquisición y el grado de conocimiento que tiene el escritor de las estrategias que intervienen en la composición de un texto, así como del control que ejerce sobre estas para optimizar su proceso de producción. Estos aportes coinciden con los datos de otros trabajos en los que se pone de manifiesto que la enseñanza de estrategias de composición favorece el aprendizaje de la escritura (Wakabayashi, 2013; Valencia y Caicedo, 2015), y en los que las aportaciones entre compañeros ayudan a mejorar las propias producciones escritas (Paquette, 2009; Lam, 2010; Guzmán y Rojas-Drummond, 2012). Además, los hallazgos encontrados en este trabajo ilustran los beneficios que la enseñanza de las estrategias de composición en grupos interactivos ofrece a la mejora de las producciones textuales. Uno de los motivos de estos logros puede deberse a las relaciones que se producen entre los procesos de oralidad y escritura entre los distintos alumnos, lo que puede resultar una medida eficaz para fomentar la reflexión individual y colectiva, así como mejorar la adquisición de estrategias de aprendizaje de la escritura. 
En cuanto al género, señalar que no se encontraron diferencias en el aprendizaje de la escritura ni en relación al proceso codificador ni en la composición textual, lo que cuestiona la necesidad de desarrollar didácticas diferenciales según el género.

Resulta de gran relevancia que conclusiones como las señaladas en el presente estudio se trasladen a los futuros docentes, ya que, tal y como señalan Birello y Gil (2014), la concepción que estos tienen de la composición escrita se encuentra todavía centrada en los modelos más tradicionales de la producción textual.

Los datos hallados en esta investigación se encuentran en la línea de otros estudios que indican que un gran número de estudiantes no han adquirido todavía las habilidades necesarias para el desarrollo de los procesos de escritura (Zumbrunn y Brunning, 2012; Méndez y Zalapa, 2013), de ahí la importancia que presenta la puesta en práctica de programas de enseñanza de estrategias de aprendizaje de composición escrita desde los primeros cursos escolares, ya que se puede experimentar una mejora considerable en los procesos de producción textual si se implementan programas de instrucción centrados en el uso de estrategias de composición (Hurtado, 2013).

En este sentido, la comunicación dialógica constituye un medio de gran valía para la mejora de la composición escrita, ya que posibilita un incremento de las interacciones alrededor de las actividades de escritura, favoreciendo la interacción entre los procesos de oralidad y escritura, a la vez que ofrece la oportunidad de practicar estilos constructivos de comunicación que amplían el repertorio lingüístico y la generación de ideas más creativas, conocimiento que permitirá al escritor identificar, seleccionar y utilizar las estrategias más adecuadas para mejorar las propias producciones textuales.

Mediante propuestas didácticas de carácter dialógico se posibilita hacer explícitas las estrategias que tanto el profesor como los propios compañeros van utilizando para construir la composición del texto. La verbalización de las estrategias que se produce en los grupos interactivos mediante las aportaciones de los distintos compañeros a través de la reflexión individual y colectiva puede considerarse una medida eficaz para la adquisición de estrategias de aprendizaje de la escritura.

En resumen, este trabajo es relevante para la mejora de la composición escrita, por lo que a nivel práctico se sugiere el diseño de programas orientados al desarrollo de las estrategias de composición, en concreto hacia aquellas que contribuyan a la consideración de los elementos de la situación de comunicación; a la generación y organización de ideas; a la estructuración general del texto, y a la revisión de la producción textual a través de grupos interactivos que potencien la puesta en común, el análisis reflexivo y el pensamiento en voz alta. Una limitación de este trabajo es que no se realizó un seguimiento para evaluar si los resultados se mantienen en el tiempo, por lo que una cuestión relevante para futuros estudios debería ser incluir medidas de seguimiento que permitan comprobar si los efectos de las intervenciones efectuadas presentan continuidad en los niveles superiores o si, por el contrario, carecen de continuidad y se desvanecen. 
RAÚL GUTIÉRREZ-FRESNEDA Y ANTONIO DÍEZ-MEDIAVILLA

EFECTOS DE LA COMUNICACIÓN DIALÓGICA EN LA MEJORA DE LA COMPOSICIÓN ESCRITA...

\section{REFERENCIAS BIBLIOGRÁFICAS}

ARROYo, R. y SALVAdOR, F. (2005) El proceso de planificación en la composición escrita de alumnos de Educación Primaria. [The planning process in written composition of pupils in primary education]. Revista de Educación, 336, 353-376.

ARTiles, C. y JiMÉneZ, J. E. (2007) PROESCRI Primaria. Prueba de evaluación de los procesos cognitivos en la escritura. [Primary PROESCRI. Assessment of cognitive processes in writing]. Consejería de Educación, Cultura y Deportes del Gobierno de Canarias.

Bereiter, C. y Scardamalia, M. (1987) Psychology of written composition. Hillsdale N. J., Lawrence Erlbaum.

BirelLo, M. y Gil JuAN, M. R. (2014) Creencias sobre la enseñanza de la composición escrita y de la gramática de estudiantes de grado de Educación Primaria y de Educación infantil. [Beliefs about teaching written composition and grammar grade students of Primary Education and Child Education]. Tejuelo, 10, 11-26.

CABERO, J.; FERnÁNDEZ, J. M. y ORTA, I. (2015) Dificultades de lectura y escritura en alumnos con nacimiento prematuro. Construcción de un instrumento de diagnóstico. [Difficulties reading and writing in children with premature birth. Construction of a diagnostic tool]. Revista de Evaluación Educativa, 4 (1).

CASTElló, M. y Monereo, C. (1996) Un estudio empírico sobre la enseñanza y el aprendizaje de estrategias para la composición de textos argumentativos. Infancia y Aprendizaje, 74, 39-55.

Coulson, D. y Harvey, M. (2013) Scaffolding student reflection for experience-based learning: A framework. Teaching in Higher Education, 18 (4), 401-413. http://dx.doi.org/ 10.1080/13562517.2012.752726.

Cuetos, F.; RAmOs, J. L. y RuANO, E. (2003) PROESC. Evaluación de los procesos de escritura. [PROESC. Evaluation of writing processes]. Madrid: TEA.

Dockrell, J. E.; Lindsay, G.; Connelly, V. y MACKIE, C. (2007) Constraints in the production of written text in children with specific language impairments. Exceptional Children, 2 (73), 147-164. http://dx.doi.org/10.1177/001440290707300202.

EsPinO, S. (2015) La enseñanza de estrategias de escritura y comunicación de textos científicos y académicos a estudiantes de posgrado. Revista Mexicana de Investigación Educativa, 20 (66), 959-976.

Flower, L. y HAYES, J. (1981) A cognitive process theory of writing. College Composition and Communication, 32, 365-87. http://dx.doi.org/10.2307/356600.

Galve, J. L. (2005) BECOLE. Batería de evaluación cognitiva de la lectura y la escritura. [Battery cognitive assessment of reading and writing]. Madrid, EOS.

Gallego, J. L. y GonZÁlez, J. (2008) Cómo planifican la composición escrita alumnos con sobredotación intelectual. [How to plan the written composition intellectually gifted students]. Revista de Investigación Educativa, 26 (2), 463-484.

Gallego, J. L. y Mendías, A. M. (2012) ¿Qué saben los estudiantes universitarios sobre la planificación de tareas de escritura? Un estudio de caso. [What college students know about planning writing assignments? A case study]. Revista de Investigación en Educación, 10 (2), 47-61.

García, J. N. y Marbán, J. M. (2003) El proceso de composición escrita en alumnos con DA y/O BR: estudio instruccional con énfasis en la planificación. Infancia y Aprendizaje, $26(1), 97-113$.

GONZÁleZ, M. J. y MARTín, I. (2006) Análisis del rendimiento en composición escrita y sus dificultades en Educación Secundaria. [Analysis of performance in written composition 
RAÚl GUTIÉRREZ-FRESNEDA Y ANTONIO DÍEZ-MEDIAVILLA

EFECTOS DE LA COMUNICACIÓN DIALÓGICA EN LA MEJORA DE LA COMPOSICIÓN ESCRITA...

and difficulties in Secondary Education]. Infancia y Aprendizaje, 29 (3), 315-326. http:// dx.doi.org/10.1174/021037006778147926.

GONZÁLEZ, R. M. (2003) Una propuesta de intervención en los procesos cognitivos y estructuras textuales en niños con DAE. [A proposal for intervention in cognitive processes and textual structures in children with DAE]. Psicothema, 15 (3), 458-463.

GuTIÉRREZ, R. (2014) La composición escrita de textos narrativos en alumnos sordos de educación secundaria. [The written narrative composition on deaf high school students]. Aula Abierta, 42, 22-27. http://dx.doi.org/10.1016/s0210-2773 (14)70004-6.

GuZMán, K. y RoJAS-Drummond, S. M. (2012) Escritura colaborativa en alumnos de primaria. Un modo social de aprender juntos. [Collaborative writing in elementary students. A social way to learn together]. Revista Mexicana de Investigación Educativa, 17 (52), 217-245.

HARRIS, K. R.; Graham, S. y MASON, L. H. (2006) Improving the wraiting, Self-Regulated Strategy Development With and Without Peer Support. American Educational Research Journal, 43 (2), 295-340.

HAYES, J. R. (1996) A new framework for understanding cognition and affect in writing, en LeVy, C. y RansDell, S. (eds.) The science of writing. Theories, methods, Individual Differences, and Applications. Mahwah, NJ: Erlbaum.

HurTADO, R. (2013) Regulación metacognitiva y composición escrita: su relación con la calidad de educación en la educación básica primaria. [Metacognitive Regulation and written composition: its relationship with the quality of education in primary basic education]. Uni-pluri/versidad, 13 (2), 35-43.

KiuHara, S.; O'Neill, R.; HAWKen, L. y Graham, S. (2012) The effectiveness of teaching 10th grade students STOP, AIMS and DARE for planning and drafting persuasive text. Exceptional Children, 78 (3), 335-355. http://dx.doi.org/10.1177/001440291207800305.

LAM, R. (2010) A Peer Review Training Workshop: Coaching Students to Give and Evaluate Peer Feedback. TESL Canada Journal, 27 (2), 114-127. http://dx.doi.org/10.18806/tesl. v27i2.1052.

MÉNDEZ, A. y ZALAPA, M. (2013) Escribir y reescribir, una posibilidad para mejorar el aprendizaje: Reporte de una experiencia con niños y niñas P'urhépecha. [Write and rewrite, a possibility to improve learning: Report of an experience with children P'urhépecha]. Uaricha, 10 (21), 97-110.

Montanero, M.; Lucero, M. y FernándeZ, M. J. (2014) Iterative co-evaluation with a rubric of narrative texts in Primary Education/Coevaluación iterativa con rúbrica de textos narrativos en la Educación Primaria. Infancia y Aprendizaje, 37 (1), 184-220. http:// dx.doi.org/10.1080/02103702.2014.881653.

OECD (2013) PISA 2012 Results: What students know and can do. Student performance in mathematics, reading and science (vol. 1). Recuperado de: http://www.oecd.org/pisa/ keyfindings/pisa-2012-results.htm.

PaqueTte, K. (2009) Integrating the 6+1 writing traits model with cross-age tutoring: an investigation of elementary students' writing. Literacy Research and Instruction, 48, 28-38. http://dx.doi.org/10.1080/19388070802226261.

Quinton, S. y Smallbone, T. (2010) Feeding forward: Using feedback to promote student reflection and learning a teaching model. Innovations in Education and Teaching International, 47 (1), 125-135. http://dx.doi.org/10.1080/14703290903525911.

Salvador, F. (2008) Psicopedagogía de la lengua escrita. Madrid, EOS. 
SAlvador, F. y García, A. (2009) El proceso de revisión en la composición escrita de alumnos de Educación Primaria. [The review process in written composition of pupils in primary education]. Revista Española de Pedagogía, 242, 61-77.

TroiA, G. A. (2006) Writing instruction for students with learning disabilities, en MacARTHuR, C. A.; Graham, S. y Fitzgerald, J. (eds.) Handbook of writing research. New Cork, Guilford Press, 324-336.

VALENCIA, M. y CAICEDO, A. M. (2015) Intervención en estrategias metacognitivas para el mejoramiento de los procesos de composición escrita. Estado de la cuestión. [Intervention metacognitive strategies to improve written composition processes. State of the question]. Revista CES Psicología, 8 (2), 1-30.

Wakabayashi, R. (2013) The Effects of the Peer Feedback Process on Reviewers' Own Writing. English Language Teaching, 6 (9), 177-192. http://dx.doi.org/10.5539/elt. v6n9p177.

WATANABE, L. y HALL-KenYON, K. (2011) Improving young children's writing: the influence of story structure on kindergartner's writing complexity. Literacy Research and Instruction, 50, 272-293. http://dx.doi.org/10.1080/19388071.2010.514035.

YARROw, F. y TOPPING, K. (2001) Collaborative writing: The effects of metacognitive prompting and structured peer interaction. British Journal of Educational Psychology, 71, 261-282.

ZuMBRUNN, S. y BRUNNING, R. (2012) Improving the writing knowledge of emergent writers: the effects of self-regulated strategy development. Reading and Writing, 25 (5), 1-20. http://dx.doi.org/10.1007/s11145-012-9384-5. 
